\title{
Angiogenesis precedes cardiomyocyte migration in regenerating mammalian hearts
}

\author{
Arnar B. Ingason, BS, ${ }^{\mathrm{a}, \mathrm{b}}$ Andrew B. Goldstone, MD, PhD, ${ }^{\mathrm{b}}$ Michael J. Paulsen, MD, ${ }^{\mathrm{b}}$ \\ Akshara D. Thakore, MSc, ${ }^{\mathrm{b}}$ Vi N. Truong, BS, ${ }^{\mathrm{b}}$ Bryan B. Edwards, BE, ${ }^{\mathrm{b}}$ Anahita Eskandari, BS, \\ Tanner Bollig, ${ }^{\mathrm{b}}$ Amanda N. Steele, MSc, ${ }^{,}$and Y. Joseph Woo, $\mathrm{MD}^{\mathrm{b}}$
}

\section{ABSTRACT}

Objective: Although the mammalian heart's ability to fully regenerate is debated, its potential to extensively repair itself is gaining support. We hypothesized that heart regeneration relies on rapid angiogenesis to support myocardial regrowth and sought to characterize the timeline for angiogenesis and cell proliferation in regeneration.

Methods: One-day-old CD-1 mice (P1, N = 60) underwent apical resection or sham surgery. Hearts were explanted at serial time points from 0 to 30 days postresection and analyzed with immunohistochemistry to visualize vessel ingrowth and cardiomyocyte migration into the resected region. Proliferating cells were labeled with 5-ethynyl-2'-deoxyuridine injections 12 hours before explant. 5Ethynyl-2'-deoxyuridine-positive cells were counted in both the apex and remote areas of the heart. Masson's trichrome was used to assess fibrosis.

Results: By 30 days postresection, hearts regenerated with minimal fibrosis. Compared with sham surgery, apical resection stimulated a significant increase in proliferation of preexisting cardiomyocytes between 3 and 11 days after injury. Capillary migration into the apical thrombus was detected as early as 2 days postresection, with development of mature arteries by 5 days postresection. New vessels became perfused by 5 days postresection as evidenced by lectin injection. Vessel density and diameter significantly increased within the resected area over 21 days, and vessel ingrowth always preceded cardiomyocyte migration, with coalignment of most migrating cardiomyocytes with ingrowing vessels.

Conclusions: Endothelial cells migrate into the apical thrombus early after resection, develop into functional arteries, and precede cardiomyocyte ingrowth during mammalian heart regeneration. This endogenous neonatal response emphasizes the importance of expeditious angiogenesis required for neomyogen-

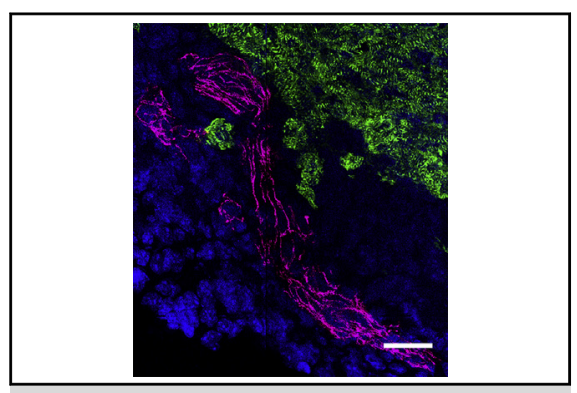

Arteriogenesis precedes cardiomyocyte migration in regeneration after apical resection.

\section{Central Message}

After apical resection in neonatal mice, angiogenesis and arteriogenesis precede cardiomyocyte migration in the newly forming apex of regenerating hearts.

\section{Perspective}

Mounting evidence in recent years suggests that neonatal murine hearts have a robust regenerative potential. Angiogenesis is a vital part of the regenerative process and could be a future therapeutic target for myocardial infarction or heart failure. We characterize the timeline for angiogenesis and cell proliferation in regenerating mammalian hearts.

See Editorial Commentary page 1128. esis. (J Thorac Cardiovasc Surg 2018;155:1118-27)

\footnotetext{
From the a Department of Medicine, University of Iceland, Reykjavík, Iceland; and ${ }^{\mathrm{b}}$ Department of Cardiothoracic Surgery, Stanford University School of Medicine, Stanford, Calif.

This work was supported by the National Institutes of Health (R01 HL089315-01 to Y.J.W.) and the American Heart Association (14POST20380744 to A.B.G.).

Read at the 97th Annual Meeting of The American Association for Thoracic Surgery, Boston, Massachusetts, April 29-May 3, 2017.

Received for publication May 1, 2017; revisions received Aug 11, 2017; accepted for publication Aug 23, 2017.

Address for reprints: Y. Joseph Woo, MD, Department of Cardiothoracic Surgery, Stanford University, Falk Building CV-235, 300 Pasteur Dr, Stanford, CA 94305-5407 (E-mail: joswoo@stanford.edu).

$0022-5223 / \$ 36.00$

Copyright (c) 2017 by The American Association for Thoracic Surgery

https://doi.org/10.1016/j.jtcvs.2017.08.127
}

Hearts of lower vertebrates, such as zebrafish and newts, have robust regenerative potential. ${ }^{1-3}$ However, mammalian cardiomyocytes have long been thought to

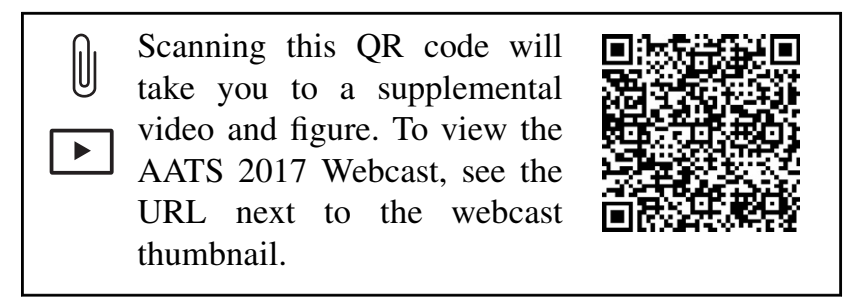




\section{Abbreviations and Acronyms \\ EdU = 5-ethynyl-2-deoxyuridine \\ FGF $=$ fibroblast growth factor \\ GFP = green fluorescent protein \\ HBSS = Hanks' balanced salt solution \\ MMP = matrix metalloprotease \\ PBS = phosphate-buffered saline \\ PBS-Tx $=$ phosphate-buffered saline containing TritonX-100 \\ $\mathrm{SDF}=$ stromal-derived factor}

permanently exit the cell cycle shortly after birth with concurrent loss of proliferation, a process known as "terminal differentiation." Further cardiac growth is provided by hypertrophy and not hyperplasia. ${ }^{4}$

Within the first week of life, mammalian cardiomyocytes replicate their DNA and become binucleated. ${ }^{5}$ Concurrently, the foramen ovale closes with atrial septation. ${ }^{6}$ Before this, the mammalian heart resembles the single circulation of the mononucleated hearts of adult zebrafish. This resemblance has led scientists to hypothesize that mammalian hearts have conserved regenerative potential in early life. Although highly controversial, ${ }^{7,8}$ recent findings suggest that neonatal mice regenerate their heart during the first week of life if exposed to injury in the form of apical resection or myocardial infarction., 90

Reestablishing blood flow to an ischemic territory of the heart after myocardial infarction is essential and may be a major limitation of tissue-engineered and cell-based therapies. ${ }^{11-}$ ${ }_{13}$ Prevascularization of ischemic myocardium before cell transplantation has been shown to increase transplant survival. ${ }^{14} \mathrm{~A}$ better understanding of the processes driving angiogenesis in neonatal heart regeneration may facilitate novel therapies for revascularization of ischemic myocardium.

Aurora and colleagues ${ }^{15}$ recently demonstrated that macrophage depletion impaired cardiac angiogenesis after myocardial infarction and that affected hearts failed to regenerate despite preserved cardiomyocyte proliferation. Additional work in nerve regeneration found that bridging capillaries guide Schwann cells to reapproximate severed axons. ${ }^{16}$ This suggests that cardiac angiogenesis may be necessary for cardiac regeneration; new vessels are likely required to support new cardiomyocytes and may even guide cardiomyocytes to the site of injury. In the present study, we confirm the regenerative potential of the neonatal murine heart and investigate the timeframe for angiogenesis after apical resection.

\section{MATERIALS AND METHODS \\ Animal Handling}

All experiments described in this study conformed to the "Guide for the Care and Use of Laboratory Animals,' published by the US National
Institutes of Health (8th ed, 2011) under the supervision of the Stanford Administrative Panel on Laboratory Animal Care (protocol 28921), Stanford's Institutional Animal Care and use Committee, which is accredited by the Association for the Assessment and Accreditation of Laboratory Animal Care.

\section{Apical Resection}

Resections of the ventricular apex of neonatal mice were performed as described previously. ${ }^{17}$ Briefly, 1-day-old CD-1 mice (P1), delivered from pregnant CD-1 mice (Charles River Laboratories, Wilmington, Mass), were subjected to apical resection or sham surgery (Video 1). With the use of ice, neonates were anesthetized by hypothermic circulatory arrest, which induces apnea and asystole, preventing excessive blood loss. Neonates were then placed supine under an operating microscope (Zeiss, Oberkochen, Germany), and the chest was sterilized with povidone-iodine (Betadine, Purdue Products LP, Stamford, Conn). A left anterolateral thoracotomy was performed at the fifth intercostal space to expose the ventricular apex. Further exposure was achieved by applying gentle pressure on the abdomen, thus pushing the heart to the exterior of the chest cavity. With the use of microscissors, the apex along with the overlying pericardium was gradually resected over several incisions until the left ventricular cavity was exposed, leaving a thin sheet of myocardium between the left ventricular cavity and the exterior. This thin cell sheet and the hyperthermia-induced circulatory arrest alleviate the concern for air embolism or need for manual compression to achieve hemostasis. The chest wall and skin were closed in separate layers with interrupted 6-0 polypropylene suture. Sham controls underwent anterolateral thoracotomy without cardiac resection. Animals were then rapidly rewarmed and cleaned of residual blood or Betadine to minimize the risk of maternal cannibalization. In addition, neonates were removed and returned to their mother as an entire litter to further reduce the risk of maternal cannibalization or neglect. The operative survival for the procedure was $88 \%$.

\section{Heart Explants}

Neonates were euthanized via hypothermia followed by decapitation. P5 and older mice were anesthetized with isoflurane before decapitation. After decapitation, the chest cavity was exposed via median sternotomy and the heart was explanted.

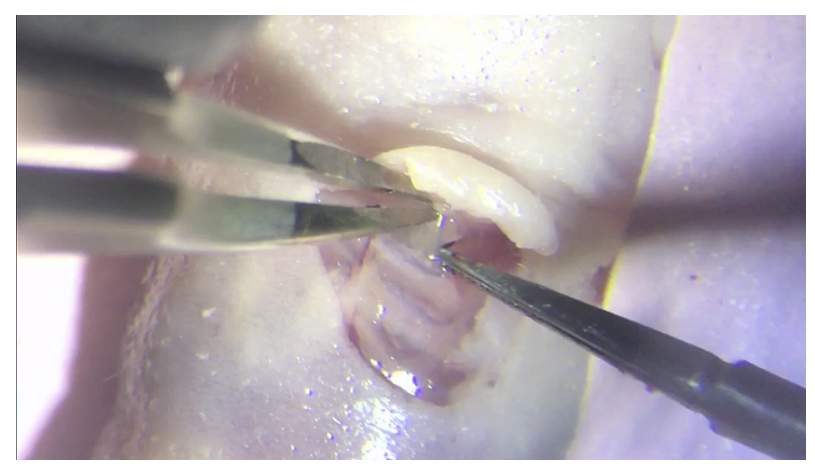

VIDEO 1. Apical resection was performed on 1-day-old neonates. Neonates were placed supine under an operating microscope, the chest was sterilized, and a left anterolateral thoracotomy was performed at the fifth intercostal space to expose the ventricular apex. By using microscissors, the apex was gradually resected until the left ventricular cavity was exposed. Before resection, neonates were anesthetized using ice to induce hypothermic circulatory arrest and prevent exsanguination. Video available at: http://www.jtcvsonline.org/article/S0022-5223(17) 31902-5/fulltext. 


\section{In Vivo Injections}

For cell proliferation measurements, 5-ethynyl-2-deoxyuridine (EdU) labeling was performed using Click-iT Imaging Kit (Invitrogen, Thermo Fisher Scientific, Waltham, Mass) per the manufacturer's instructions. Briefly, EdU was injected intraperitoneally 12 hours before cardiac explant. EdU positivity was assessed with immunohistochemistry.

For assessment of vessel perfusion, lectin conjugated to a far-red fluorophore was injected into the right ventricle of anesthetized mice 2 minutes before decapitation and heart explantation to allow for adequate circulation of lectin.

\section{Immunostaining}

After heart explantation, specimens were rinsed with phosphatebuffered saline (PBS) and embedded in optimum cutting temperature freezing medium before being frozen in dry ice; $10-\mu \mathrm{m}$ sagittal sections were then created using a cryostat (Cryo Star NX70, Thermo Fisher Scientific).

Sections were fixed in $4 \%$ paraformaldehyde for 10 minutes, permeabilized in PBS containing 0.5\% Tween for 15 minutes, and then blocked in $10 \%$ fetal bovine serum for 1 hour at room temperature. Next, primary antibodies diluted in PBS were added and incubated for 90 minutes at $37^{\circ} \mathrm{C}$. The following primary antibodies were used for immunohistochemistry staining at the indicated dilutions: $\alpha$-smooth muscle actin at 1:200 (ab5694, ab5694, and ab7817; Abcam plc, Cambridge, United Kingdom), sarcomeric $\alpha$-actinin at 1:200 (ab9465, Abcam), troponin I at 1:200 (ab56357, Abcam), and vascular endothelial cadherin at 1:600 (ab33168, Abcam). Appropriate secondary antibodies diluted in PBS at 1:200 were then added and incubated for 45 minutes at $37^{\circ} \mathrm{C}$. Finally, nuclear staining was performed using DAPI (Thermo Fisher Scientific) or Hoechst 33342 (Thermo Fisher Scientific). Sections were washed in PBS 3 times between each step. EdU labeling was performed using the Click-iT Imaging Kit (Thermo Fisher Scientific) according to the manufacturer's instructions.

For whole mount staining, neonatal hearts were explanted, rinsed with PBS, and then put into $4 \%$ paraformaldehyde for 1 hour before being washed 3 times in PBS containing $0.2 \%$ Triton X-100 (phosphate-buffered saline containing TritonX-100 [PBS-Tx]) for 10 minutes. Primary antibodies diluted in $0.2 \%$ PBS-Tx were added and allowed to incubate on a rocker at $4{ }^{\circ} \mathrm{C}$ for 48 hours. Thereafter, hearts were washed 3 times in $0.2 \%$ PBS-Tx for 10 minutes each and then every 2 hours for the next 6 hours. The following day, secondary antibodies, diluted in $0.2 \%$ PBS$\mathrm{Tx}$, were added and allowed to incubate on a rocker at $4^{\circ} \mathrm{C}$ for 48 hours. Subsequently, hearts were washed 3 times in $0.2 \%$ PBS-Tx and every 2 hours thereafter for the rest of the day. The next day, hearts were put in Vectashield (Vector Laboratories, Burlingame, Calif) and imaged.

\section{Masson's Trichrome Staining}

Frozen sections were acquired as described previously. Sections were thawed for 20 minutes at $4{ }^{\circ} \mathrm{C}$ and then rinsed in $100 \%$ ethanol before being fixed in Bouin's solution for 3 hours at room temperature. Excess stain was rinsed off in running water until water ran clear. Slides were stained in Biebrich Scarlet-Acid Fuchsin before being washed in running water for 2 minutes. Thereafter, slides were stained in phosphotungstic/ phosphomolybdic acid solution for 15 minutes and Aniline Blue solution for 20 minutes. Next, slides were placed in $1 \%$ acetic acid solution for 5 minutes before being rinsed in running water for 2 minutes. Last, slides were dehydrated in $95 \%$ ethanol, $100 \%$ ethanol, and xylene for 2 minutes each. Cytoseal 60 (Thermo Fisher Scientific) was used to attach slides to cover slips.

\section{Confocal Imaging and Analysis}

Confocal images were obtained using a Zeiss LSM 780 confocal microscope and Zen imaging software. The area of the resected apex was imaged using the tile scan feature, using the same acquisition settings and number of z-stacks for each sample and experiment. Maximum intensity projections of the z-stacks were acquired using Fiji software.

Blood vessel, smooth muscle, and fibrotic area measurements were obtained using Fiji software. For vessel and smooth muscle measurements, the area of apical thrombus was selected and the signal of interest isolated. Images were converted to 8-bit greyscale TIFF, thresholded, made binary, and measured using the measurement function. For measuring fibrosis, the apical area was first selectively cropped; next, the whole apical area and fibrotic-only area were independently measured with the color threshold function. Apical thrombosis percentage was calculated by dividing the size of the fibrotic area by the total area of the heart.

\section{Cardiomyocyte Isolation and Cardiomyocyte/ Endothelial Cell Co-Culture}

Cardiomyocyte isolation was performed using the Pierce Primary Cardiomyocyte Isolation Kit (Thermo Fisher Scientific) according to the manufacturer's instructions. Briefly, hearts from P1-P3 mice that constitutively express green fluorescent protein (GFP) under the chicken beta-actin promoter were explanted under sterile conditions. Atria were removed from the explanted hearts and the ventricles placed in $500 \mu \mathrm{L}$ ice-cold Hanks' balanced salt solution (HBSS). Ventricles were then minced into 1- to $3-\mathrm{mm}^{3}$ pieces using scissors and washed in ice-cold HBSS twice for 5 minutes, being centrifuged briefly at full speed between washes. Thereafter, HBSS was aspirated and $200 \mu \mathrm{L}$ of digestive enzyme solution 1 , containing papain, and $10 \mu \mathrm{L}$ of digestive solution 2 , containing thermolysin, were added. Cells were allowed to incubate at $37^{\circ} \mathrm{C}$ for 35 minutes before being washed twice for 5 minutes in ice-cold HBSS, being briefly centrifuged at full speed between washes. HBSS was aspirated, and Dulbecco's Modified Eagle Media containing 10\% fetal bovine serum was added. Cell yield and viability were determined using Trypan Blue staining with a hemocytometer. Next, a 24-well tissue culture plate was coated with $100 \mu \mathrm{L}$ of Matrigel per well and allowed to incubate at $37^{\circ} \mathrm{C}$ for 1 hour. Next, 25,000 human umbilical vein endothelial cells were plated onto the Matrigel and incubated for 12 hours at $37^{\circ} \mathrm{C}$ to allow tubules to form. Next, 25,000 GFP-positive cardiomyocytes were seeded onto the tubules. Images were acquired every 10 minutes over 12 hours using time-lapse microscopy.

\section{Statistical Analysis}

All data are presented as mean \pm standard error of the mean unless otherwise specified. The Wilcoxon test was used to compare differences between 2 groups. The Kruskal-Wallis test was used to compare difference between multiple groups. The Conover test with Bonferroni correction was used as a post hoc test. All statistical tests were 2-tailed.

\section{RESULTS \\ Murine Heart Regenerates With Minimal Fibrosis}

After apical resection, a thrombus quickly forms to seal the cut myocardium and exposed ventricular cavity, preventing exsanguination. Over time, the thrombus organizes and by 11 days postresection becomes fibrotic. Gradually, the fibrotic area is replaced by myocardium; by 30 days postresection the heart has regenerated with minimal fibrosis (Figure 1, A). Quantification of the apical fibrotic area demonstrates that from 11 to 30 days postresection, apical fibrosis reduces significantly $(3.37 \% \pm 0.48 \%$ vs $1.03 \% \pm 0.40 \%$, respectively, $P=.010)$ (Figure $1, B)$. Sham hearts $(0.14 \% \pm 0.03 \%)$ were significantly less fibrotic than hearts 11 days postresection $(3.37 \% \pm 0.48 \%, P=.002)$ and 14 days 


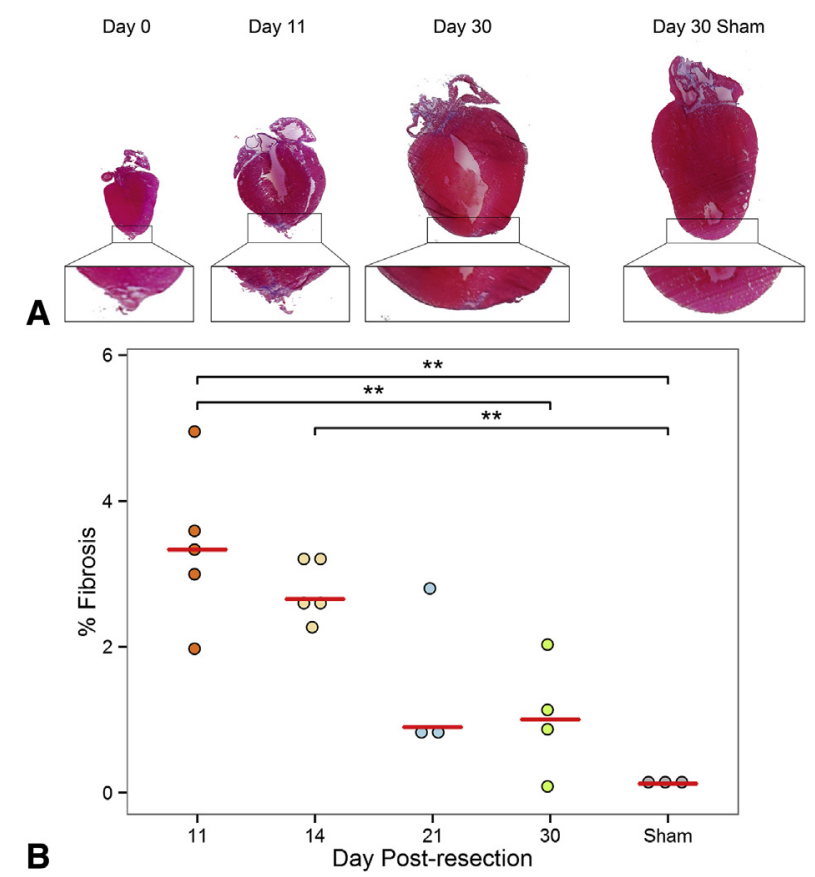

FIGURE 1. The neonatal heart regenerates with minimal fibrosis after apical resection. A, Masson's trichrome staining of hearts at 0,11 , and 30 days postresection. For comparison, sham-operated hearts, harvested 30 days after surgery, are included. A magnified image of each apex is provided below. An apical thrombus quickly formed after resection and became fibrotic by 11 days postresection. By 30 days postresection, the heart had regenerated with minimal fibrosis. B, Quantification of fibrotic area in the apex after apical resection at 11, 14, 21, and 30 days postresection. For comparison, sham-operated hearts harvested 30 days after surgery are included. Values are represented as percentage fibrosis of the apical area. Fibrosis in the apex consistently decreased over time. This suggests that fibrosis is gradually replaced by myocardium as heart regeneration progresses. Red bars represent median value for each timepoint. Asterisks denote statistical significance at $* * P<.01$.

postresection $(2.78 \% \pm 0.19 \%, P=.010)$; however, no significant difference in fibrosis was noted between sham hearts and hearts explanted 21 and 30 days postresection. $(1.49 \% \pm 0.66 \%$ and $1.03 \% \pm 0.40 \%$, respectively) (Figure 1, B). To measure cell proliferation, EdU injections were performed 12 hours before explant to label proliferating cells within the heart. EdU-positive cells were counted in both the apex and remote areas of the heart. To see if resected hearts were more proliferative than sham controls, the number of EdU-positive cells was compared between the 2 groups. Although no difference was noted at 2 days postresection between animals treated with apical resection and sham surgery, animals 3 to 11 days postresection demonstrated a sustained increase in proliferative cardiomyocytes (Figure 2, $A$ and $B$ ). Measurements of proliferating endothelial cells revealed that $32 \%$ of the migrating endothelial cells were EdU positive.

\section{Vessels Migrate Into the Apical Thrombus and Become Functional Early}

To visualize revascularization of the apex after resection and to see if blood vessel ingrowth precedes cardiomyocyte migration, immunohistochemical staining was performed and vessel density measurements of the resected region were performed using Fiji software. Numerous sagittal sections of hearts from 0 to 21 days postresection were analyzed. Migrating vessels were first observed 2 days postresection within the apical thrombus (Figure 3, A). Arteries, characterized by a surrounding smooth muscle cell layer, developed within the thrombus in 1 of 5 hearts by 5 days postresection (Figure 3, B). By 7 days postresection and onward, all resected hearts contained true smooth muscle cell-supported arteries in the region of thrombus (Figure 3, C). Experiments with lectin perfusion revealed that blood vessels in the resected region become functional by 5 days postresection (Figure $3, D$ ). By using whole mount staining, capillary plexus formation at 7 days postresection was observed in the apical thrombus, characteristic of vessel remodeling (Figure E1). Together, endothelial cells invade the apical thrombus early and grow into functional arteries over time as part of the regenerative response.

After apical resection, vascular density of the apical thrombus continuously increased over time (Figure 4, A). Likewise, smooth muscle density increased over time, with significant increase from $0.12 \% \pm 0.09 \%$ at 5 days postresection to $2.19 \% \pm 0.83 \%$ at 11 days postresection $(P=.019)$ (Figure $4, B$ and $D)$. Last, maximal vessel diameter increased over time, with significant increase from $13.45 \pm 3.45 \mu \mathrm{m}^{2}$ at 5 days postresection to $65.70 \pm 10.27 \mu \mathrm{m}^{2}$ at 11 days postresection $(P=.043)$ (Figure 4, $C$ and $D$ ).

\section{Vessel Ingrowth Precedes Cardiomyocyte Migration}

Migrating cardiomyocytes, characterized by cellular protrusions or processes, ${ }^{18}$ were first observed at 2 days postresection. Of note, vessel ingrowth preceded cardiomyocyte migration in all sections. In fact, most migrating cardiomyocyte processes were observed along the border of migrating blood vessels (Figure 5, A). For quantification, the distance between the furthest part of a cardiomyocyte process and the nearest vessel in the apical thrombus was measured. Measurements from 215 cardiomyocyte processes showed that $69 \%$ of cardiomyocyte processes were within $15 \mu \mathrm{m}$ of the nearest vessel, a distance representing the average diameter of 2 nuclei. The average distance between a cardiomyocyte process and its nearest blood vessel was $12.4 \pm 0.9 \mu \mathrm{m}$. This proximity was replicated in vitro by co-culturing GFP-positive murine cardiomyocytes and human umbilical vein endothelial cells, which demonstrated that the majority 


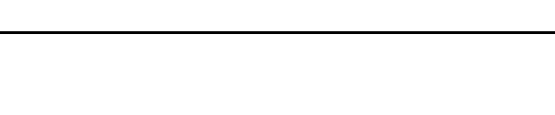
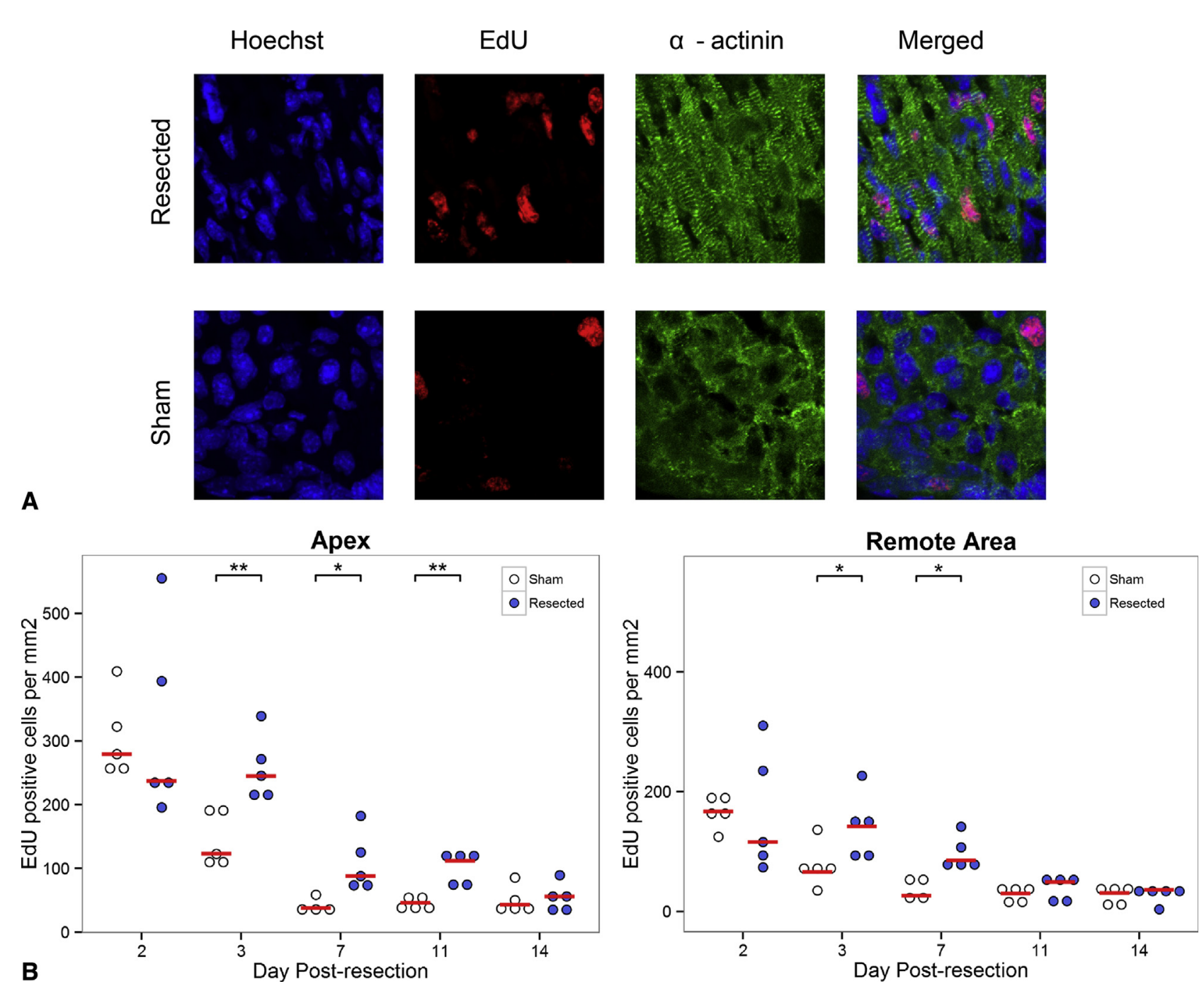

FIGURE 2. Apical resection prolongs window of cardiomyocyte proliferation. A, Proliferative cardiomyocytes were identified by staining for $\alpha$-actinin (green), EdU (red), and Hoechst 33342 (blue). The number of EdU-positive cells in the apex 3 days after operation is compared between sham and apical resection groups. EdU staining represents cardiomyocytes that have gone through at least 1 cell cycle of replication. B, EdU measurements comparing the number of proliferating cells in the apex and remote myocardium of resected hearts and sham controls at each time point. Cardiomyocyte proliferation is increased relative to sham controls at 3 days postresection in both the apex and remote area of the heart compared with sham-operated animals and is sustained for over 1 week in the apex. Red bars represent median value for each timepoint. Asterisks denote statistical significance at $* P<.05$ and $* * P<.01 . E d U, 5$-Ethynyl-2-deoxyuridine.

of cardiomyocytes co-aligned with the endothelial network (Figure 5, B).

\section{DISCUSSION}

Our findings support previous reports that the heart regenerates after resection. ${ }^{9}$ Although highly controversial and publicly debated, ${ }^{7,8}$ the heart may regenerate completely after apical resection. ${ }^{9,10}$ Although we noted some fibrosis in hearts 30 days postresection, the amount of apical fibrosis did not significantly differ from sham hearts. Therefore, despite this limited fibrosis, our results do suggest that the regenerative potential of the neonatal heart is real and robust. Furthermore, cell proliferation was increased relative to sham controls from 3 to 11 days postresection. This suggests that the stimulus for regeneration does not augment cardiomyocyte proliferation beyond the standard immediate postnatal rate. Rather, the stimulus sustains high levels of proliferation for 1 week beyond that of normal postnatal development. Immunohistochemical staining showed that angiogenesis preceded cardiomyocyte migration in the apical thrombus. Additionally, the vascularity, maximal vessel diameter, and arterial maturation significantly increased within the resected region over time. Capillary formation in the apical thrombus was first observed at 2 days postresection and arteries at 5 days postresection. 

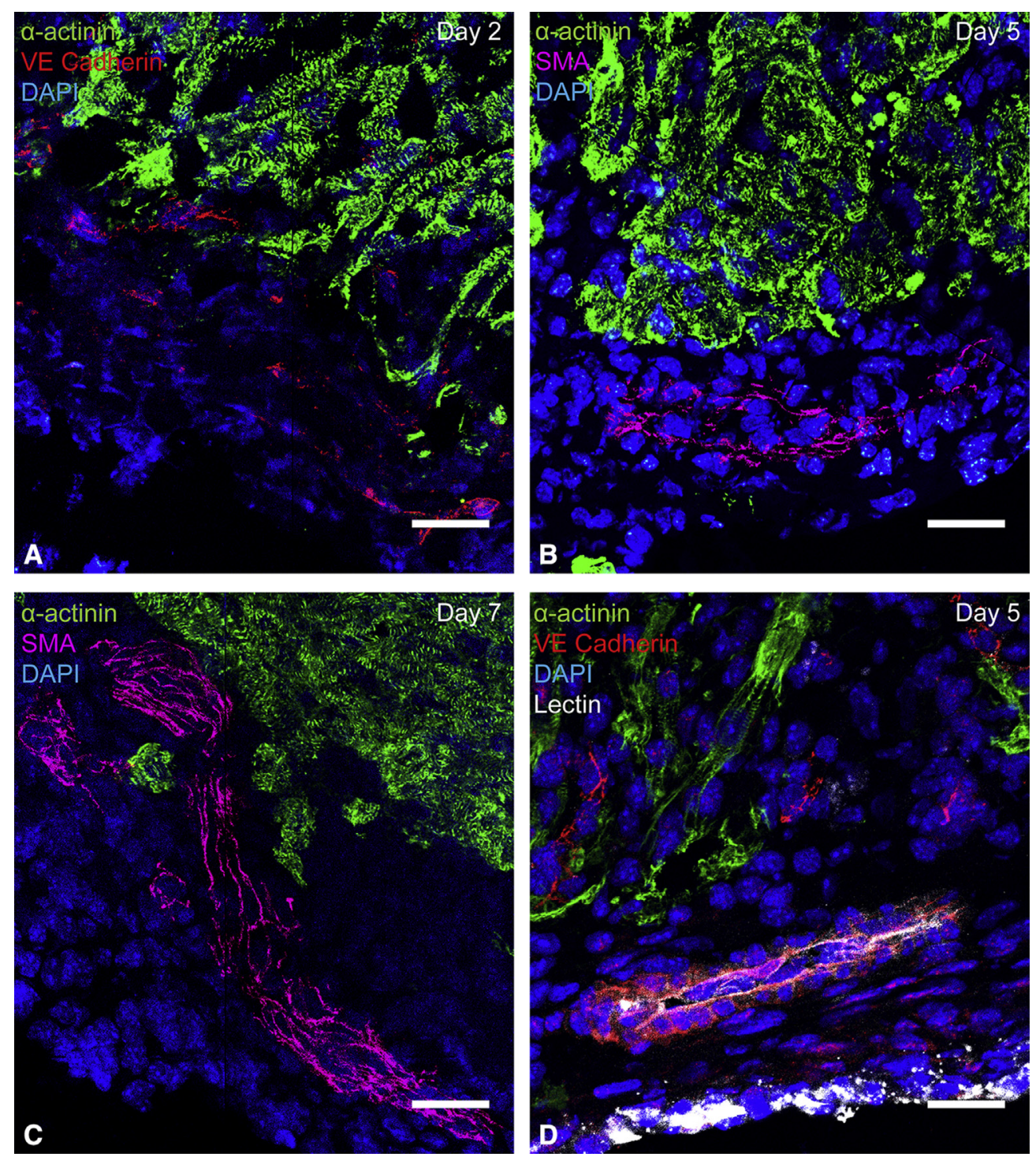

FIGURE 3. Vessel ingrowth into the apical thrombus precedes cardiomyocyte migration after resection. Histologic analysis of sagittal heart sections demonstrated that vessels enter the apical thrombus early and mature over time. Endothelial cells always extended deeper into the thrombus than cardiomyocytes. A, Migrating endothelial cells in the apical thrombus, visualized by vascular endothelial cadherin staining. Capillary formation was first noted in the apical thrombus at 2 days postresection. B, An artery characterized by a smooth muscle layer in the apical thrombus at 5 days postresection. Arteries in the apical thrombus were first noted at 5 days postresection, but did not consistently appear until 7 days postresection. C, A large ingrowing artery within the apical thrombus at 7 days postresection. Arteries were found within apical thrombus of all hearts treated with apical resection at 7 days postresection and onward. D, A lectin-perfused vessel in the apical thrombus at 5 days postresection. This suggests that ingrowing vessels become functional by 5 days postresection. Green $=\alpha$-actinin, $r e d=$ vascular endothelial cadherin, red, magenta $=$ smooth muscle actin, blue $=$ DAPI, white $=$ lectin. All scale bars represent $25 \mu \mathrm{m}$. SMA, Smooth muscle actin; VE, vascular endothelial; DAPI, 4',6-diamidino-2-phenylindole.

The formation of larger arteries, characterized by a surrounding smooth muscle layer, within the apical thrombus by 5 days postresection is suggestive of de novo arteriogenesis. Microvessels appeared to remodel to form larger vessels. Blood vessels are already perfused within 5 days postresection, suggesting that increased blood flow might stimulate vessel remodeling. This is consistent with arteriogenesis after arterial occlusion, where sheer stress from increased blood flow may contribute to collateral artery remodeling. ${ }^{19}$ In addition, hemodynamic forces in the mouse yolk-sac have been shown to be both sufficient and necessary for vessel maturation. ${ }^{20}$

The fact that angiogenesis precedes cardiomyocyte migration is consistent with findings in other organ systems. Recent studies using a rat and mouse model have shown that peripheral nerve regeneration after sciatic nerve transection is dependent on macrophage-induced angiogenesis between the 2 cut ends of a transected nerve. The newly formed 


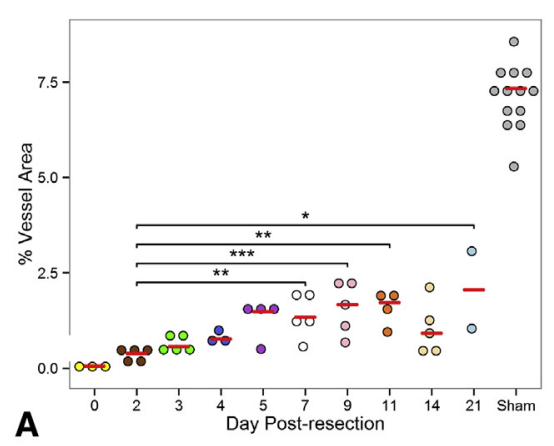

A
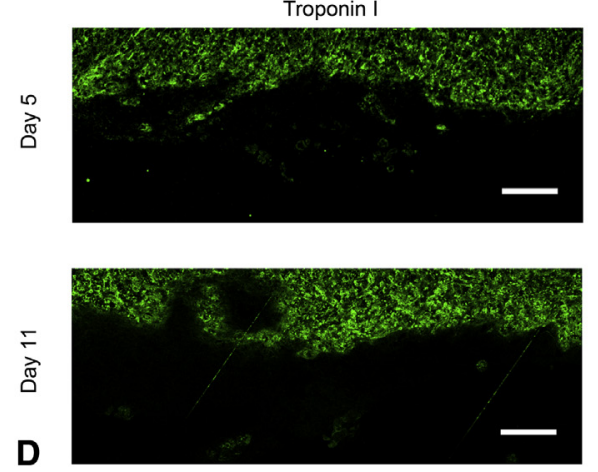

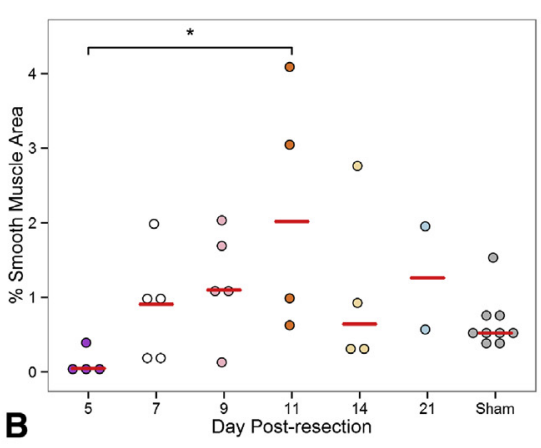

SMA
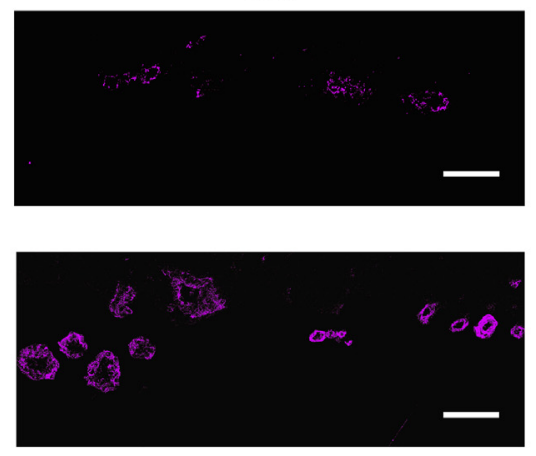

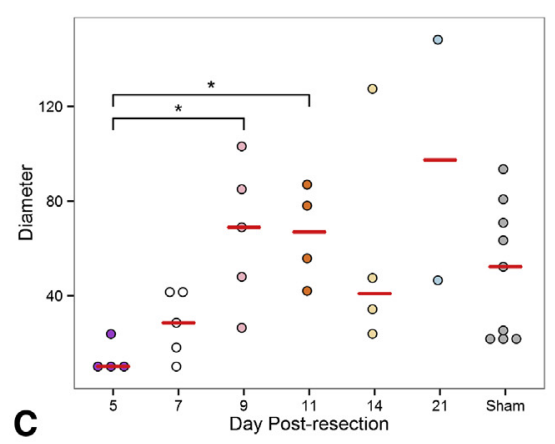

Merged
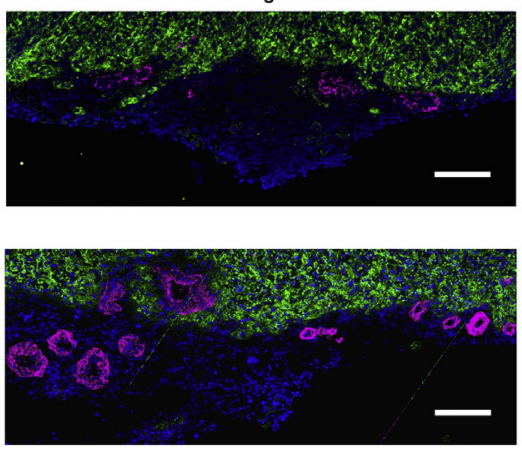

FIGURE 4. The apical thrombus becomes more vascularized over time to ultimately regrow apical coronary arteries. A, Vascular density of apical thrombus consistently increased from the time of resection to 9 days postresection, after which it plateaued. B, Percentage of smooth muscle cell area in the apical thrombus. C, Maximal vessel diameter in the apical thrombus. For comparison, measurements of apices of sham-operated hearts are provided for all graphs. D, Comparison of arterial formation in the apical thrombus of resected hearts 5 and 11 days postresection. Note the increased arterial density and vessel diameter at 11 days postresection compared with 5 days postresection. Green $=$ troponin I, magenta $=$ smooth muscle actin, blue $=$ DAPI. Scale bar represents $25 \mu \mathrm{m}$. Red bars represent median value for each timepoint. Asterisks denote statistical significance at $* P<.05, * * P<.01$, and $* * * P<.001$. SMA, Smooth muscle actin.

vessels form a bridge between the stumps, which Schwann cells use as a physical guide to regenerate axons. Whether heart regeneration follows a similar pathway is unknown. By using immunochemical staining, we showed that the majority of migratory cardiomyocytes are in close proximity to endothelial cells. Our estimates of the relationship between cardiomyocyte processes and endothelial cells are limited by the fact that $10 \mu \mathrm{m}$-thick sections were used for staining. Whole mount staining might further clarify whether all migratory cardiomyocytes are found along growing vessels.

The mechanisms governing neoangiogenesis and cardiomyocyte migration in neonatal mice after apical resection still remain to be elucidated. A study using ventricular amputation in zebrafish demonstrated that epicardial-derived stromal-derived factor (SDF)- $1 \alpha$ induces cardiomyocyte migration. ${ }^{21}$ Of note, the SDF- $1 \alpha$ receptor CXCR4b was expressed in cardiomyocytes but not endothelial cells. Furthermore, blocking the CXCR4b receptor resulted in defective cardiomyocyte migration without affecting endothelial cells. SDF- $1 \beta$, a different isoform expressed in zebrafish but not mammals, directs endothelial cells during coronary artery formation in zebrafish. $^{21,22}$ Therefore, the close proximity of migrating cardiomyocytes and blood vessels that we observed could be due to the presence of a common chemoattractant, such as SDF- $1 \alpha$, which stimulates both endothelial and cardiomyocyte migration. In fact, transgenic mice with a fluorescent reporter knocked into 1 SDF- $1 \alpha$ allele demonstrate that arterial endothelial cells strongly express SDF- $1 \alpha .{ }^{23}$ Because cardiomyocytes and arterial endothelial cells both express CXCR4, it is possible that arterial endothelial cell-derived SDF- $1 \alpha$ guides a cascade of sprouting angiogenesis, arteriogenesis, and cardiomyocyte migration after myocardial injury. This may also explain the close proximity of migratory cardiomyocytes and endothelial cells noted in our study.

The mechanism and cell source of the blood vessels that invade the apical thrombus postresection remain unknown. Studies have shown that bone marrow-derived stem cells contribute to neovascularization through paracrine signaling, but do not directly incorporate into the endothelial layer. ${ }^{2-27}$ Another possible source for endothelial progenitors in the heart is the epicardium. Epicardial cells have been shown to undergo epithelial-to-mesenchymal transition and contribute to neovascularization of the apical thrombus. ${ }^{28}$ However, whether the epicardium is a richenough source of progenitors to reconstitute the majority of the vasculature is unlikely; the most probable candidate cells for repopulation of the apical vasculature are 

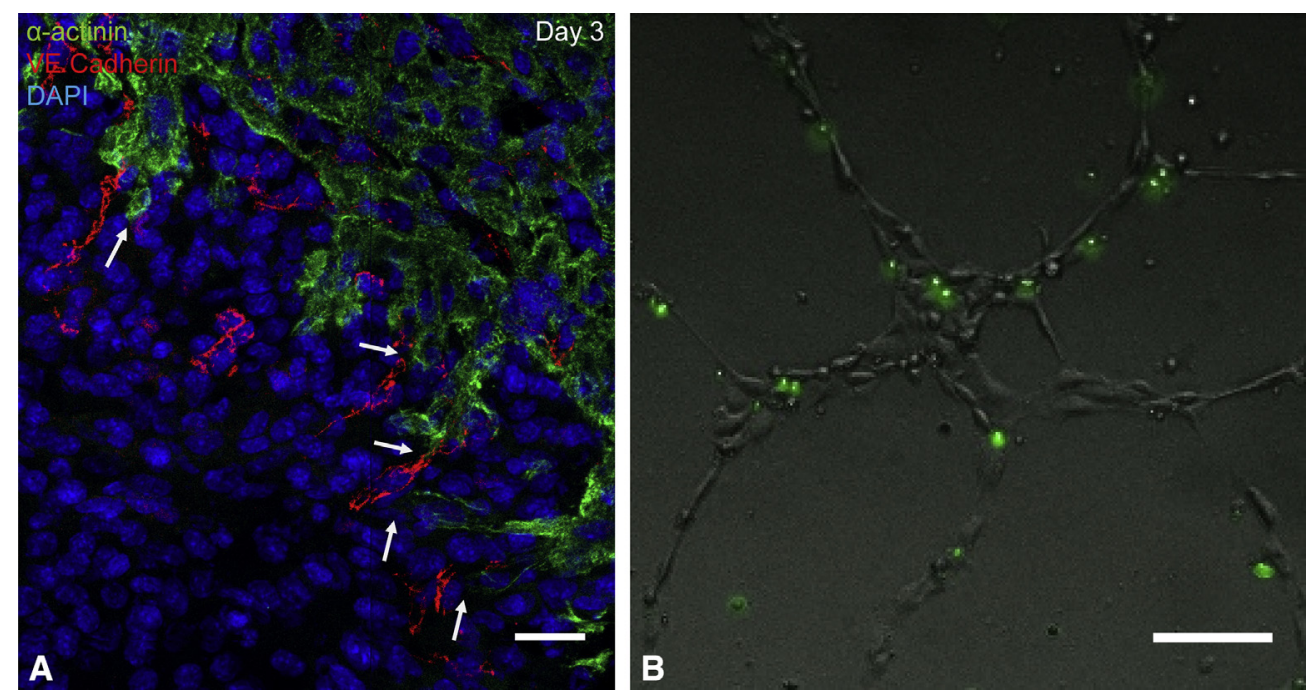

FIGURE 5. Migratory cardiomyocytes in the regenerating apex are in close proximity to endothelial cells. A, Immunohistochemical staining of apical thrombus 3 days postresection. After apical resection, endothelial cells migrate into the apical thrombus ahead of cardiomyocytes. Most migrating cardiomyocytes were observed in close proximity to ingrowing vessels. Note the close proximity of cardiomyocyte processes to migrating vessels in the apical thrombus (arrows). Green $=\alpha$-actinin, red $=$ vascular endothelial cadherin, blue $=$ DAPI. Scale bars represent $25 \mu \mathrm{m}$. B, In vitro co-culturing of murine GFP-positive cardiomyocytes and human umbilical vein endothelial cells. Nearly all cardiomyocytes co-align with the endothelial network. Scale bars represent $25 \mu \mathrm{m}$. Together, this suggests that a strong relationship exists between the 2 cell types. VE, Vascular endothelial; DAPI, 4',6-diamidino-2phenylindole.

preexisting endothelial cells. Lineage-traced coronary endothelial cells in zebrafish reconstituted the vasculature in the neo-apex after ventricular amputation. ${ }^{29}$ Our analysis of cell proliferation demonstrated that a large number of endothelial cells within the apical thrombus are proliferative and suggests that preexisting endothelial cells may also repopulate the vasculature in mice after myocardial injury. However, a similar lineage-tracing study in neonatal mice is required to better confirm this process.

After initial vascularization in embryos, larger vessels form a perivascular mural layer in a process termed "arteriogenesis." Studies using arterial ligations in mice and rabbits have demonstrated collateral vessels form from remodeling preexisting arterioles, and not by capillary maturation. ${ }^{19,30,31}$ In apical resection models, preexisting vasculature is removed; therefore, arteries found within the apical thrombus are likely derived from maturation and remodeling of invading capillaries.

Our findings suggest that the heart is still proliferative during the first days of life and that myocardial injury does not augment postnatal proliferation but rather prolongs the initial proliferative period. Alternatively, the stimulus for cardiomyocyte proliferation might not be initiated until 2 to 3 days after injury, potentially because the cells that induce cardiomyocyte proliferation are not found within the apical thrombus until then. These might be activated epicardial cells, inflammatory cells, such as macrophages, or bone marrow-derived stem cells, all of which have been shown to play a key role in heart regeneration. ${ }^{15,32,33}$

\section{Study Limitations}

Our results must be interpreted in the context of several limitations. The model of apical resection may differ significantly from that of myocardial infarction, because tissue and underlying extracellular matrix are removed in apical resection rather than left in situ as in myocardial infarction. This may directly affect the extent of the local inflammatory response. Whether our results can be applied to revascularization and regeneration after myocardial infarction is unknown. Furthermore, our data are obtained from histologic sections of hearts explanted at different time points; without in vivo microscopy, we cannot observe regeneration in the same heart over time.

\section{CONCLUSIONS}

Our findings provide insight into the process by which the heart regenerates in neonates and confirms the heart's transient regenerative potential. Reestablishing the vasculature is paramount to not only support cardiomyocytes but also guide them to the area of injury. Mimicking this support and guidance may be important for the success of cellbased therapies focused on remuscularizing infarcted myocardium or even therapies focused on in situ reprogramming. Whether similar modes of repair can be induced after myocardial infarction, however, remains to be determined. 


\section{Webcast}

You can watch a Webcast of this AATS meeting presentation by going to: https://aats.blob.core.windows.net/ media/17AM/2017-05-02/RM310/05-02-17_Room310_ 0740_Ingason.mp4.

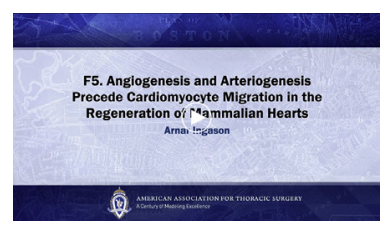

\section{Conflict of Interest Statement}

Authors have nothing to disclose with regard to commercial support.

\section{References}

1. Poss KD, Wilson LG, Keating MT. Heart regeneration in zebrafish. Science. 2002;298:2188-90

2. Witman N, Murtuza B, Davis B, Arner A, Morrison JI. Recapitulation of developmental cardiogenesis governs the morphological and functional regeneration of adult newt hearts following injury. Dev Biol. 2011:354:67-76.

3. Oberpriller JO, Oberpriller JC. Response of the adult newt ventricle to injury. $J$ Exp Zool. 1974;187:249-59.

4. Tam SKC, Gu W, Mahdavi V, Nadal-Ginard B. Cardiac myocyte terminal differentiation. Ann N Y Acad Sci. 1995;752:72-9.

5. Li FQ, Wang XJ, Capasso JM, Gerdes AM. Rapid transition of cardiac myocytes from hyperplasia to hypertrophy during postnatal development. J Mol Cell Cardiol. 1996:28:1737-46.

6. Cole-Jeffrey CT, Terada R, Neth MR, Wessels A, Kasahara H. Progressive anatomical closure of foramen ovale in normal neonatal mouse hearts. Anat Rec (Hoboken). 2012;295:764-8.

7. Andersen Ditte C, Ganesalingam S, Jensen Charlotte H, Sheikh Søren P. Do neonatal mouse hearts regenerate following heart apex resection? Stem Cell Rep. 2014;2:406-13.

8. Sadek HA, Martin JF, Takeuchi JK, Leor J, Nei Y, Giacca M, et al. Multi-investigator letter on reproducibility of neonatal heart regeneration following apical resection. Stem Cell Rep. 2014;3:1.

9. Porrello ER, Mahmoud AI, Simpson E, Hill JA, Richardson JA, Olson EN, et al. Transient regenerative potential of the neonatal mouse heart. Science. 2011;331: 1078-80.

10. Porrello ER, Mahmoud AI, Simpson E, Johnson BA, Grinsfelder D, Canseco D, et al. Regulation of neonatal and adult mammalian heart regeneration by the miR15 family. Proc Natl Acad Sci U S A. 2013:110:187-92.

11. Mathison M, Gersch RP, Nasser A, Lilo S, Korman M, Fourman M, et al. In vivo cardiac cellular reprogramming efficacy is enhanced by angiogenic preconditioning of the infarcted myocardium with vascular endothelial growth factor. $J \mathrm{Am}$ Heart Assoc. 2012:1:e005652.

12. Gaffey AC, Chen MH, Venkataraman CM, Trubelja A, Rodell CB, Dinh PV, et al. Injectable shear-thinning hydrogels used to deliver endothelial progenitor cells, enhance cell engraftment, and improve ischemic myocardium. J Thorac Cardiovasc Surg. 2015;150:1268-76.

13. MacArthur JW, Goldstone AB, Cohen JE, Hiesinger W, Woo YJ. Cell transplantation in heart failure: where do we stand in 2016? Eur J Cardiothorac Surg. 2016;50:396-9.

14. Sakakibara Y, Nishimura K, Tambara K, Yamamoto M, Lu F, Tabata Y, et al. Prevascularization with gelatin microspheres containing basic fibroblast growth factor enhances the benefits of cardiomyocyte transplantation. J Thorac Cardiovasc Surg. 2002;124:50-6.

15. Aurora AB, Porrello ER, Tan W, Mahmoud AI, Hi JA, Bassel-Duby R, et al. Macrophages are required for neonatal heart regeneration. J Clin Invest. 2014;124 1382-92.

16. Cattin A-L, Burden Jemima J, Van Emmenis L, Mackenzie Francesca E, Hoving Julian JA, Garcia Calavia N, et al. Macrophage-induced blood vessels guide schwann cell-mediated regeneration of peripheral nerves. Cell. 2015;162 1127-39.

17. Mahmoud AI, Porrello ER, Kimura W, Olson EN, Sadek HA. Surgical models for cardiac regeneration in neonatal mice. Nat Protoc. 2014;9:305-11.

18. Morikawa Y, Zhang M, Heallen T, Leach J, Tao G, Xiao Y, et al. Actin cytoskeletal remodeling with protrusion formation is essential for heart regeneration in Hippo-deficient mice. Sci Signal. 2015;8:ra41.

19. Schaper W. Collateral circulation. Basic Res Cardiol. 2009;104:5-21.

20. Lucitti JL, Jones EAV, Huang C, Chen J, Fraser SE, Dickinson ME. Vascular re modeling of the mouse yolk sac requires hemodynamic force. Development. 2007:134:3317-26.

21. Itou J, Oishi I, Kawakami H, Glass TJ, Richter J, Johnson A, et al. Migration of cardiomyocytes is essential for heart regeneration in zebrafish. Development. 2012; 139:4133-42.

22. Harrison Michael RM, Bussmann J, Huang Y, Zhao L, Osorio A, Burns CG, et al. Chemokine-guided angiogenesis directs coronary vasculature formation in zebrafish. Dev Cell. 2015;33:442-54.

23. Chang AH, Raftrey BC, D'Amato G, Surya VN, Poduri A, Chen HI, et al DACH1 stimulates shear stress-guided endothelial cell migration and coronary artery growth through the CXCL12-CXCR4 signaling axis. Genes Dev. August 4, 2017 [Epub ahead of print].

24. Rehman J, Li J, Orschell CM, March KL. Peripheral blood "endothelial progen itor cells" are derived from monocyte/macrophages and secrete angiogenic growth factors. Circulation. 2003;107:1164-9.

25. Rajantie I, Ilmonen M, Alminaite A, Ozerdem U, Alitalo K, Salven P. Adult bone marrow-derived cells recruited during angiogenesis comprise precursors for periendothelial vascular mural cells. Blood. 2004;104:2084-6.

26. Ziegelhoeffer T, Fernandez B, Kostin S, Heil M, Voswinckel R, Helisch A, et al Bone marrow-derived cells do not incorporate into the adult growing vasculature. Circ Res. 2004;94:230-8.

27. Pearson JD. Endothelial progenitor cells—hype or hope? J Thromb Haemost. 2009;7:255-62

28. Lepilina A, Coon AN, Kikuchi K, Holdway JE, Roberts RW, Burns CG, et al. A dynamic epicardial injury response supports progenitor cell activity during zebrafish heart regeneration. Cell. 2006;127:607-19.

29. Zhao L, Borikova AL, Ben-Yair R, Guner-Ataman B, MacRae CA, Lee RT, et al. Notch signaling regulates cardiomyocyte proliferation during zebrafish heart regeneration. Proc Natl Acad Sci. 2014;111:1403-8.

30. Scholz D, Ito W, Fleming I, Deindl E, Sauer A, Wiesnet M, et al. Ultrastructure and molecular histology of rabbit hind-limb collateral artery growth (arteriogen esis). Virchows Arch. 2000;436:257-70.

31. Wagner S, Helisch A, Ziegelhoeffer T, Bachmann G, Schaper W. Magnetic resonance angiography of collateral vessels in a murine femoral artery ligation model. NMR Biomed. 2004;17:21-7.

32. Masters M, Riley PR. The epicardium signals the way towards heart regeneration. Stem Cell Res. 2014;13:683-92.

33. Loffredo Francesco S, Steinhauser Matthew L, Gannon J, Lee Richard T. Bone marrow-derived cell therapy stimulates endogenous cardiomyocyte progenitors and promotes cardiac repair. Cell Stem Cell. 2011;8:389-98.

Key Words: angiogenesis, arteriogenesis, cardiac regeneration, cardiomyocyte migration, vascular biology

\section{Discussion}

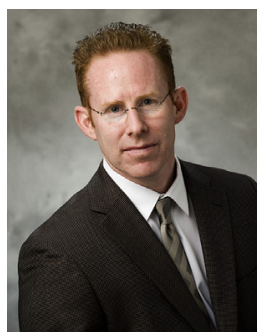

Dr B. Leshnower (Atlanta, Ga). Arnar, congratulations on a very elegant study and a well-written manuscript, and I really liked the video. I love the fact that you are performing circulatory arrest on mice. I have 3 questions, and will ask them one at a time.

In your experiments, the increase in cardiomyocyte proliferation in the apical resection animals occurred in a delayed fashion, day 3 to 11 . Why do you 
think there was that 72-hour delay in cell regeneration after your initial resection?

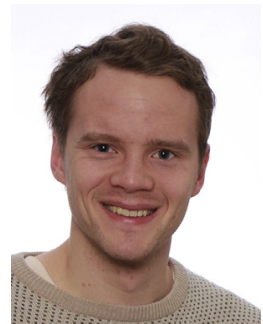

Mr Arnar B. Ingason (Stanford, Calif). The 72-hour delay was interesting and suggests that the neonatal heart is still proliferative during the first days of life and that myocardial injury actually prolongs this proliferative period without augmenting the initial proliferative response. Alternately, the cells that induce cardiomyocyte proliferation could migrate the apical thrombus after day 2 or 3, and thus the stimuli for cardiomyocyte proliferation might not occur until that time.

Dr Leshnower. In the article, you hypothesize that SDF1-alpha might be involved in the mechanism, in the arteriogenesis and cardiomyocyte migration. Do you have any further thoughts on what the source of the SDF-1alpha is and its overall role in guiding the apical regeneration?

Mr Ingason. SDF-1-alpha is a potent chemoattractant that has been shown in apical resection in the zebrafish model to guide cardiomyocyte migration. It is also essential for coronary artery formation in utero. Our data suggests that in the heart, SDF-1-alpha is primarily expressed in arterial endothelial cells, and the receptor for SDF-1-alpha is expressed in cardiomyocytes and arterial endothelial cells. So SDF-1-alpha might direct cardiomyocyte migration in the heart as well, and as we see, the endothelial cells are close to migrating cardiomyocytes. SDF-1-alpha might induce arteriogenesis in the thrombus area. Our results have demonstrated mixed results, with near complete inhibition of arterial formation in some animals and reduced angiogenesis with little effect in other hearts. Unfortunately, AMD3100 treatment, the inhibitor that we used, is not a perfect model, and a genetic knockout model might be necessary to further elucidate the role of SDF-1-alpha.
Dr Leshnower. The last question I have for you is based on your studies, translate this for us into a clinical scenario. How would you propose to modulate ongoing cell-based therapies to augment regeneration in patients with ischemic cardiomyopathy?

Mr Ingason. It can be argued that the major limiting factor in cell-based therapies and tissue engineering is cell survival. I think it is essential to prevascularize the ischemic area, as this may augment cell proliferation and even guide other cells into the ischemic area, and, as we reported, might even guide cardiomyocyte migration directly.

Dr Leshnower. Great work.

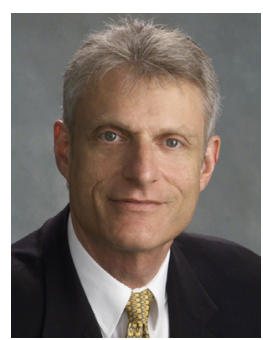

Dr P. Kurlansky (New York, NY). In terms of perspective, do you know how many days late you can do this? In other words, if you did this on day 2 , day 3, day 4 , when does it stop working? On the other hand, if you just take a normal heart, when do you stop seeing proliferation of cardiomyocytes? Even though the heart is growing, it doesn't mean that myocytes are proliferating, when the potential proliferative phase ends in mice.

Mr Ingason. The initial report by Porrello showed that the neonatal mouse heart has a proliferative window of 1 week, and if you performed apical resection on day 7 , the heart does not regenerate. Another article showed that by knocking out a specific miRNA, this proliferative period could be extended to 3 weeks. Other reports have shown that the adult mammalian heart is proliferative throughout life, but it is very little proliferation; approximately $0.5 \%$ of the cardiomyocytes are proliferative, that is not enough to repair the heart after ischemic cardiomyopathy. A possible way to augment the cardiomyocyte proliferation and increase angiogenesis is essential for future therapies. 


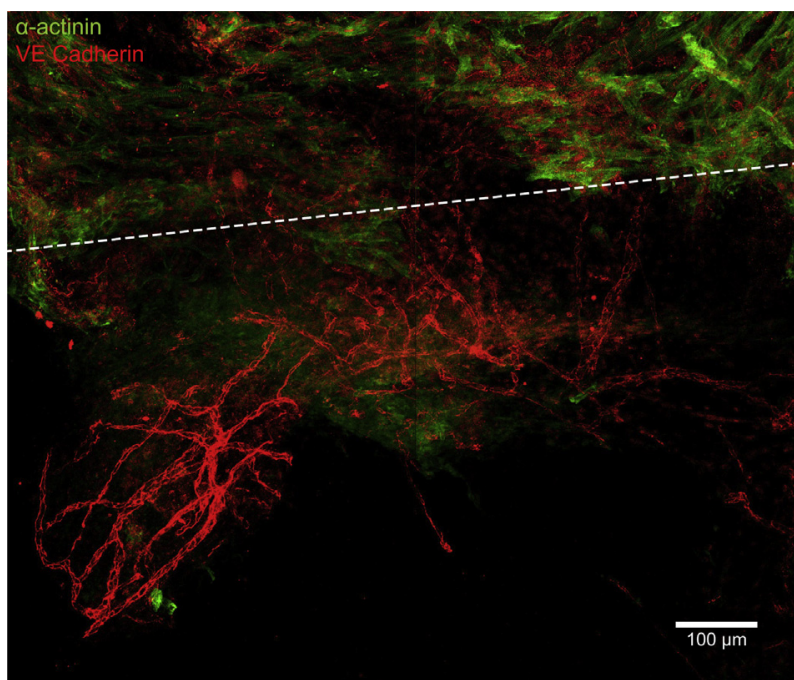

FIGURE E1. Vessel remodeling within the apical thrombus. Whole mount imaging of a heart 7 days postresection. Note the remodeling capillary plexus below the myocardial border (dotted line). Green $=\alpha$-actinin, $r e d=$ vascular endothelial cadherin. $V E$, Vascular endothelial. 